ISSN electrónico: 1885-5210

DOI: http://dx.doi.org/10.14201/rmc2020162103109

\title{
LA CHICA DANESA / THE DANISH GIRL (2015), EL DERECHO DE DECIDIR NUESTRA IDENTIDAD DE GÉNERO
}

\section{The Danish Girl (2015), the right to decide our own gender identity}

Francisco Ignacio MORETA VELAYOS; Carolina MORETA MONTERO; Olga VELASCO GUIJARRO; Inés de la FUENTE HERMOSÍN; Nieves MONTERO SÁNCHEZ; Carmen RAMÍREZ ORIVE

Centro de Salud Parque Coímbra. Gerencia de Atención Primaria. Servicio Madrileño de Salud. Móstoles. Madrid (España).

e-mail: fimoreta@yahoo.es

Fecha de recepción: 1 de julio de 2019

Fecha de aceptación: 2 de septiembre de 2019

Fecha del Avance On-Line: 3 de marzo de 2020

Fecha de publicación: 1 de junio de 2020

\section{Resumen}

Aunque el derecho a decidir nuestra propia identidad de género está reconocido como Derecho Humano Universal, las personas trans en nuestro medio siguen siendo objeto de rechazo y discriminación, lo que hace necesario el desarrollo de leyes y normas que garanticen todos nuestros derechos. En estas leyes se desarrollan múltiples aspectos relacionados con la sanidad y que los profesionales debemos integrar en nuestra actividad cotidiana en los Centros de Salud de Atención Primaria.

Partiendo desde una perspectiva histórica y el recurso de un estreno cinematográfico, fomentamos en el equipo el debate en torno a la identidad sexual, el fenómeno trans y la legislación que ampara a las personas.

Palabras clave: identidad de género; orientación sexual; transexual; transgénero; cine.

\section{Summary}

Although the right to decide our own gender identity is recognized as a Universal Human Right, trans people are still object to rejection and discrimination, which require the development of laws and regulations that guarantee our rights. In these laws are stated multiple aspects related to health and professionals should integrate them in our daily activity in General Practice Clinics.

From a historical perspective and the use of a film premiere, we encourage the debate about sexual identity, the trans phenomenon and legislation that protects people.

Keywords: gender identity; sexual orientation; transsexual; transgender; cinema.

Rev. Med. Cine. 2020; 16(2), 111-118 Ediciones Universidad de Salamanca / ॠథ® J. Med. Mov., 2020; 16 (2), $111-118$ 
LA CHICA DANESA / THE DANISH GIRL (2015), EL DERECHO DE DECIDIR NUESTRA IDENTIDAD DE GÉNERO FRANCISCO IGNACIO MORETA; CAROLINA MORETA; OLGA VELASCO; INÉS DE LA FUENTE; NIEVES MONTERO; CARMEN RAMÍREZ

Introducción

"¿Saben por qué los venecianos son tan aficionados a las máscaras? Porque dudan de su rostro de carne». Lorenzo Da Ponte ${ }^{1}$.

El estreno en los cines españoles de La chica danesa / The Danish Girl (2015) de Tom Hooper, en enero de 2016, coincidió en el tiempo con la publicación de la $L E Y 2 / 2016$, de Identidad y Expresión de Genero e Igualdad Social y no Discriminación de la Comunidad de Madrid ${ }^{2}$ y LEY $3 / 2016$, de protección integral contra la LGTBIfobia y la discriminación por razón de orientación e identidad sexual en la Comunidad de Madrid ${ }^{3}$ (En el futuro Ley de la CM).

Según la Unidad de Identidad de Género del Hospital Ramón y Cajal se estima una prevalencia en la Comunidad de Madrid de 22,1 transexuales por cada 100.000 habitantes, 31.2 para MtF (Hombre a Mujer) y 12.9 para FtM (Mujer a Hombre), ratio aproximado MtF/FtM de 2.2:1. Se trata de las cifras más altas de las publicadas por el resto de ciudades europeas y que reflejarían la accesibilidad a la unidad, la situación legal y el entorno social permisivo y abierto de España ${ }^{4}$. Estos datos, pueden servirnos para hacernos una idea de la relevancia del tema que planteamos.

La leyes de la CM disponen que «se desarrollarán e implementarán programas de capacitación, sensibilización u otros dirigidos a contrarrestar entre el personal funcionario, laboral, estatutario y sanitario de las administraciones y de los organismos, sociedades y entes públicos madrileños las actitudes discriminatorias, los prejuicios y los estereotipos dominantes por motivos de identidad de género».

Para aproximarnos a los temas relacionados con la identidad y expresión de género y facilitar la difusión y debate de las Leyes de la CM en nuestro Equipo de Atención Primaria, utilizamos el cine partiendo de la premisa de que dentro de los medios de comunicación de masas es el que con más honestidad retrata los temas minoritarios ${ }^{5}$.

\section{Aspectos conceptuales}

La diversidad terminológica (Tabla 1 ) del tema que nos ocupa puede decirse que está en proporción directa con la complejidad de la expresión sexual humana y va más allá de aspectos puramente médico-biológicos, sobre todo psicosociales $y$, admitida la diversidad sexual como un derecho humano, consideramos interesante precisar determinados conceptos tomando como punto de partida las definiciones recogidas en los Principios de Yogyakarta ${ }^{6}$ y en la propia ley de la CM como forma de unificar nuestro lenguaje.

\section{Orientación sexual}

Se trata de la capacidad de cada persona de sentir profunda atracción emocional, afectiva y sexual por personas de un género diferente al suyo (heterosexualidad), o de su mismo género (homosexualidad), o de más de un género (bisexualidad), así como la capacidad de mantener relaciones íntimas y sexuales con estas personas.

También se incluyen en éste apartado las personas asexuales o que no sienten atracción sexual alguna?.

\section{Identidad sexual / Identidad de género}

Es la vivencia interna e individual del género tal como cada persona la siente profundamente, la cual podría corresponder o no con el sexo asignado al momento del nacimiento (transgénero), incluyendo la vivencia personal del cuerpo, que podría involucrar la modificación de la apariencia - la función corporal a través de medios médicos, quirúrgicos o de otra índole (transexualismo), siempre que la misma sea libremente escogida; $y$ otras expresiones de género, incluyendo la vestimenta, el modo de hablar y los modales.

Con el prefijo trans (opuesto al prefijo cis) nos referimos a todas aquellas personas que expresan/

Rev. Med. Cine. 2020; 16(2), 111-118 Ediciones Universidad de Salamanca / ॠ@ J. Med. Mov., 2020; 16 (2), 111-118 
experimentan un género/sexo distinto al asignado al nacer y que según la ley de la CM ampara múltiples formas de expresión de la identidad de género o subcategorías como transexuales, transgénero, travestis, variantes de genero queer o personas de genero diferenciado, así como a quienes definen su género como "otro» o describen su identidad en sus propias palabras.

La intersexualidad incluye una variedad de situaciones en las cuales, una persona nace con una anatomía reproductiva o sexual que no parece encajar en las definiciones típicas de masculino y femenino.

Mientras que la transexualidad es un concepto biomédico que legitima el empleo de distintas técnicas médicas conducentes a la reasignación de género, el transgenerismo es un concepto desarrollado por las propias personas trans para desvincularse de la gestión dual biomédica y social de su género (masculino / femenino, hombre / mujer). Así la teoría queer niega que solamente existan dos sexos, ya que en la naturaleza no existen categorías binarias, solo en la mente humana ${ }^{5}$. Aunque ambos paradigmas puedan parecer opuestos será difícil encontrar a una persona que se ajuste estrictamente a los parámetros que conforman a cada uno de ellos ${ }^{8}$.

\section{Aspectos históricos y antropológicos}

Aunque hasta fechas recientes la Medicina y la Sexología han considerado las tendencias sexuales de hombres y mujeres estrictamente ligadas al sexo anatómico calificando como patológica cualquier cambio de tendencia, desde el inicio de la cultura y probablemente antes, las personas han optado por construir y reconstruir sus cuerpos como han querido o se han visto obligados ${ }^{9}$.

Son numerosas las narraciones míticas e históricas que hacen referencia cuando menos al cambio de roles de género entre sexos. En las sociedades cazadoras-recolectoras los individuos que nacían con algún tipo de intersexualidad eran vistos como signos de buen augurio. En el código de Hammurabi se menciona un tipo de mujeres denominadas "salziknrum» («hija masculina») a las que se les reconocía derechos especiales. Entre los egipcios Isis y Osiris representan la oposición y la igual naturaleza de las deidades femenina / masculina, que en ocasiones se fundían en una sola persona y cuerpo, los de Isis. Del mundo griego, en las Metamorfosis de Ovidio todos los cambios de género y cuerpo son posibles incluyendo la autofecundación (Hermafrodita, hijo de Hermes y Afrodita). Hipócrates describió entre los escitas al grupo de los no-hombres que sin estar castrados tenían ocupaciones e inclinaciones femeninas. En la Roma clásica tanto el travestismo como el transexualismo fueron duramente castigados especialmente hacia el fin del imperio y la emergencia del cristianismo; curiosamente Nerón fue travestido y legisló sobre las intervenciones quirúrgicas de cambio de sexo y para su uso personal ordenó intervenir a su esclavo Esporun para convertirlo en mujer casándose posteriormente con él ${ }^{5}$.

Los países asiáticos tienen historias centenarias de existencia de varones con variantes de género, que en los tiempos actuales habrían sido etiquetados como «mujeres transgénero». El Kama Sutra contiene elocuentes descripciones de la vida sexual de personas con «tercera naturaleza».

En la India, existen personas con una amplia gama de identidades, culturas o experiencias relacionadas con transgénero siendo las Hijras o Aravanis las más conocidas, aunque la mayoría nacieron varones, se refieren a ellas mismas en femenino y visten ropas de mujer pudiendo haber sido emasculadas o no. En el pasado fueron tratadas con gran respeto, actualmente en determinadas comunidades siguen siendo consideradas portadoras de prosperidad para la comunidad; se las invita a nacimientos y bodas en la creencia de que favorecen la fecundidad. Viven en pequeñas comunidades ganándose la vida en el servicio doméstico y eventualmente la prostitución, enfrentándose a los riesgos de la marginalidad incluidas las enfermedades de transmisión sexual ${ }^{10}$.

Rev. Med. Cine. 2020; 16(2), 111-118 Ediciones Universidad de Salamanca / @®@ J. Med. Mov., 2020; 16 (2), $111-118$ 
LA CHICA DANESA / THE DANISH GIRL (2015), EL DERECHO DE DECIDIR NUESTRA IDENTIDAD DE GÉNERO FRANCISCO IGNACIO MORETA; CAROLINA MORETA; OLGA VELASCO; INÉS DE LA FUENTE; NIEVES MONTERO; CARMEN RAMÍREZ

Tabla 1. Terminología en torno a la orientación e identidad sexual.

\begin{tabular}{|l|l|}
\hline Orientación sexual & $\begin{array}{l}\text { Heterosexualidad } \\
\text { Homosexualidad (gay, lesbiana, bull dyke) Bisexualidad } \\
\text { Asexualidad Pansexualidad }\end{array}$ \\
\hline Identidad sexual / Identidad de género Trans \\
$\begin{array}{l}\text { Metagenerismo (No binarismo, } \\
\text { genderqueer) }\end{array}$ & $\begin{array}{l}\text { Transgénero (cross-gender, gender bender) Transexual } \\
\text { Gender funck (género confuso) Andróginos } \\
\text { Intersexuales / Diversos ( neutro, tercer género, tercer sexo, hijras) } \\
\text { Queer (diferente) Transeúntes de género Genero fluido } \\
\text { Butch Otros }\end{array}$ \\
\hline Transformismo & Drags queen Drags king \\
\hline Travestismo / Crossdressers & Travestismo no fetichista Travestismo fetichista \\
\hline
\end{tabular}

Mientras redactábamos el presente artículo nos llegaba la noticia de la despenalización de la homosexualidad en la India «Soy quien soy. Así que aceptadme tal cual», dijo en la sala el presidente del Supremo, citando al escritor alemán Goethe al leer parte del fallo ${ }^{11}$.

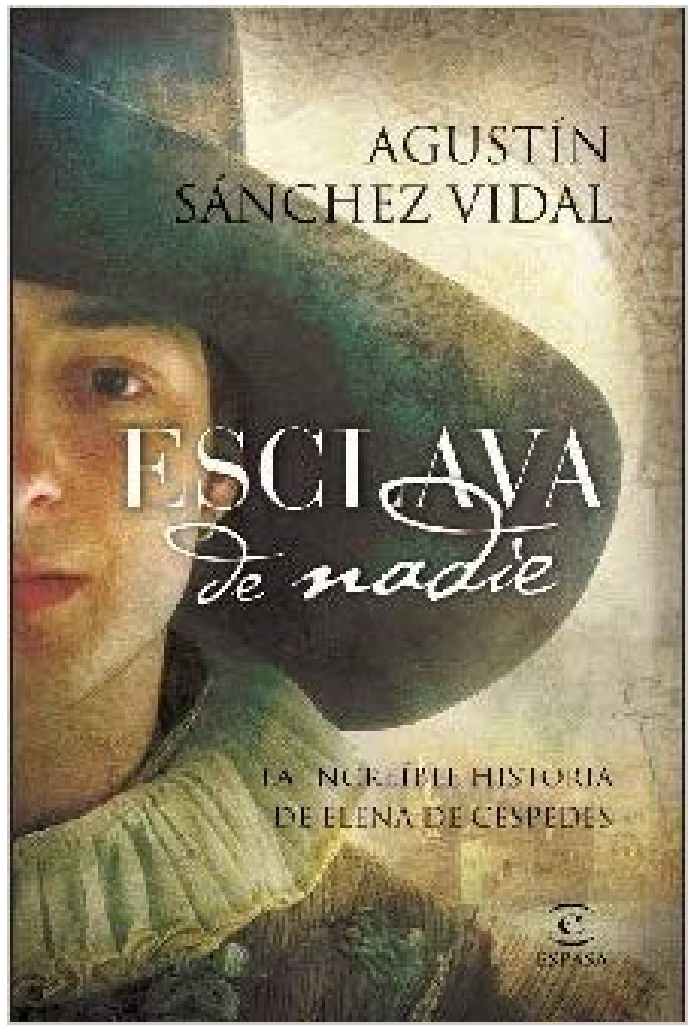

Foto 1. Portada de Esclava de nadie.
En España, entre los países de tradición judeo-cristiana, la transexualidad fue asociada con la homosexualidad y por tanto proscrita por las normas religiosas y en muchos caso criminalizada por los códigos penales hasta fechas muy recientes (Ley de Peligrosidad y Rehabilitación Social de 1970, v.g.), siendo las personas afectadas obligadas a vivir en la clandestinidad o llevadas a la hoguera a manos de la Inquisición en otros tiempos. Actualmente en la iglesia Católica el papa Francisco se manifiesta en desacuerdo con la enseñanza en los colegios de la ideología de género $^{12}$. Recientemente el arzobispo de Valencia ha considerado una "maldad» el trámite de la legislación sobre identidad de género en las Cortes Valencianas ${ }^{13}$.

Aunque el arte y la literatura españolas presentan numerosos casos de intersexualidad ( $\mathrm{La}$ mujer barbuda de José de Ribera, Italia 1631 v.g.) y transexualidad, nos parece interesante traer aquí la obra de Agustín Sánchez Vidal (Foto 1). En su novela Esclava de nadie (2010) ${ }^{14}$, relata la azarosa vida de Elen@ de Céspedes en la España de Felipe II, ampliamente documentada en el proceso inquisitorial que se llevó contra ella en Ocaña (Toledo) entre 1587 y 1588 . Nacida como mujer tuvo un hijo, después de distintas vicisitudes llegó a conseguir como hombre el título de cirujano en Madrid, vetado a las mujeres; el Dr Francisco Díaz, urólogo y médico personal de Felipe II, después de reconocerle certificó su condición de varón que le permitiría contraer matrimonio. Acusado por un 
vecino de sodomía al Santo Oficio fue finalmente encontrada culpable de sodomía, bigamia, perjurio y burla del sacramento del matrimonio y castigada a 200 azotes y a trabajar durante 10 años en un hospital sin sueldo y vestida de mujer. Aunque a día de hoy no hay acuerdo de si se trató de una hermafrodita, travestida, transexual o lesbiana, sí queda claro que representó la lucha de una persona en contra de las reglas establecidas en ese momento donde la mujer no tenía cabida en una sociedad manejada por hombres ${ }^{15}$.

\section{Del paradigma biomédico a los Derechos Humanos}

El término "transexualidad» se populariza a mediados de los años 60 del siglo XX por el endocrinólogo de origen alemán Harry Benjamin, cuyo trabajo junto al de otros coetáneos será determinante para la configuración del actual paradigma biomédico de la transexualidad y del establecimiento de protocolos para la «terapia de reasignación sexual ${ }^{9}$, si bien desde 1931 (Lili Elba) se tiene conocimiento de intervenciones quirúrgicas para el cambio de sexo y creación de neo-vaginas, siendo notorios los éxitos del Dr. Hamburger en los años 50.

Pese a que desde entonces se han ido descriminalizado las conductas trans, la transexualidad se ha considerado hasta la actualidad por la medicina como una enfermedad o trastorno, figurando como tal en el principal manual de diagnóstico psiquiátrico (DSM-5: Disforia de género) y en la Clasificación Internacional de Enfermedades (CIE10) de la $O M S^{16-17}$. Esto ha supuesto la patologización y estigmatización de las identidades de género trans junto a la paradoja de acceder a los deseos de transformación corporal mediante tratamientos médico-quirúrgicos de personas que padecen un «trastorno mental». Para otros la asunción de las tesis biologistas puede entenderse como una forma de conjurar la visión del transexual como un pervertido de un lado y de otro la de conseguir la inclusión social y registral de los afectados con la identidad sentida.

A día de hoy y en nuestro medio, el reconocimiento de que la identidad de género es un Derecho Humano Universal es sin duda el mayor avance en orden a garantizar la igualdad y el respeto a la dignidad de las personas trans. Para garantizar la aplicación universal se ha desarrollado una amplia legislación y recomendaciones que van desde la Declaración Universal de Derechos Humanos, pasando por los Principios de Yogyakarta y la resolución de la ONU de 2011 sobre «Derechos humanos, orientación sexual e identidad de género", la Carta de Derechos Fundamentales de la Unión Europea o la propia Constitución Española.

La OMS ha adoptado recientemente la CIE-11, en la que las categorías relacionadas con las personas trans han sido quitadas del Capítulo sobre Trastornos Mentales y del Comportamiento, lo cual significa que las identidades trans están formalmente des-psicopatologizadas ${ }^{18}$.

La ley de la Comunidad de Madrid es un paso más frente a la exclusión social o la violencia transfóbica aun presentes en nuestra sociedad, buscando la despatologización y promoviendo una atención médica y social integral basada en el principio de respeto a la libre manifestación de la identidad de género de los ciudadanos.

Sesión de cine en la Primaria: La chica danesa / The Danish Girl

\section{Ficha técnica}

Título: La chica danesa.

Título original: The Danish Girl.

País: Reino Unido.

Año: 2015.

Director: Tom Hooper.

Guión: Lucinda Coxon (Novela: David Ebershoff). Música: Alexandre Desplat.

Fotografía: Danny Cohen.

Montaje: William Goldenberg.

Rev. Med. Cine. 2020; 16(2), 111-118 Ediciones Universidad de Salamanca / @®@ J. Med. Mov., 2020; 16 (2), $111-118$ 
Intérpretes: Eddie Redmayne, Alicia Vikander, Matthias Schoenaerts, Amber Heard, Ben Whishaw, Sebastian Koch, Victoria Emslie, Adrian Schiller, Richard Dixon, Paul Kerry, Helen Evans, Michael Gade Thomsen, Alicia Woodhouse.

Duración: 120 minutos.

Productoras: Focus Features / Working Title Films. Género: drama; romance; años 20; transexualidad; transgénero; pintura; basado en hechos reales.

Sinopsis: «Dinamarca, años 20. La pareja de pintores formada por Einar (Eddie Redmayne) y Gerda Wegener (Alicia Vikander) disfruta de su éxito. Un día, por casualidad, la modelo a la que ella ha contratado para retratar en sus cuadros ha tenido que cancelar la cita y necesita terminar esas pinturas a tiempo. Para que ella pueda finalizar su trabajo, la pintora le pregunta a su marido si no le importaría ponerse medias y zapatos de mujer por unos instantes, a lo que él accederá sin problema. Esta revelación desencadenará el despertar de un anhelo largamente escondido por Einar: su deseo de ser mujer.

Al principio este juego les resulta apasionante y divertido, cuando los retratos resultan ser un éxito, ella anima a su marido a adoptar una apariencia femenina. Después no lo será tanto para Einar, que se da cuenta de que se siente una mujer por dentro. La vida del matrimonio dará un enorme giro y el nacimiento de una identidad femenina en el cuerpo masculino de Einar provocará muchos problemas en su relación sentimental. En su lucha por hacer justicia a su yo interior, Einar se convertirá en Lili Elbe. Su decisión final será la de hacerse una operación de cambio de sexo, convirtiéndose así en pionero en la cultura transgénero y uno de los primeros en operarse y cambiar de sexo. Este drama está basado en la verdadera historia de esta pareja de artistas daneses, protagonistas de una de las más inusuales historias de amor de principios del siglo XX» (Sensacine).

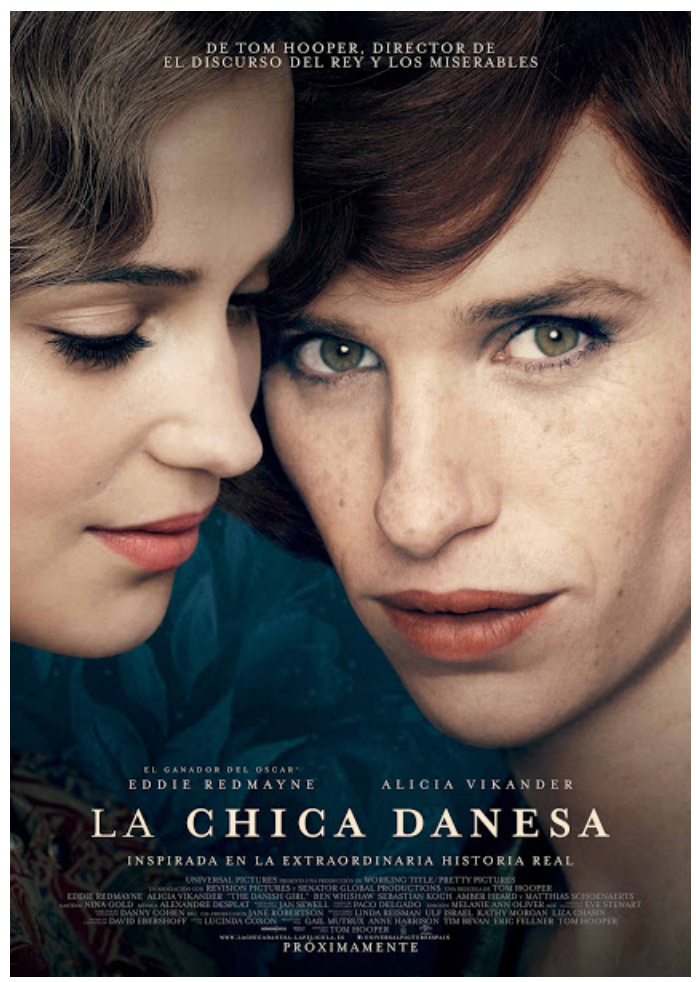

Cartel español.

\section{Enlaces:}

https://www.filmaffinity.com/es/film907970.

html

http://www.sensacine.com/peliculas/pelicula-140552

De entre la amplia filmografía que aborda el tema en cuestión y volviendo al principio, por su coincidencia en el tiempo, recurrimos a La chica danesa como catalizador para el debate sobre transexualidad en el equipo de Atención Primaria. La acogida fue favorable por los responsables de promover la difusión de la Ley de la CM ya que por otra parte algunos ya la habíamos visto, e independientemente de la crítica discrepante, encontrábamos en la misma muchos puntos clave a destacar en las sesiones que pretendíamos programar.

Rev. Med. Cine. 2020; 16(2), 111-118 Ediciones Universidad de Salamanca / 
Aunque la historia y las situaciones que nos relata se sitúan en un espacio y tiempo relativamente remotos contiene elementos y vivencias que en poco difieren de las circunstancias que actualmente toca vivir a muchas personas de condición similar en nuestro entorno, permitiendo adentrarnos en el entendimiento de la identidad de género; sufrimiento, incomprensión, discriminación o fobia, patologización, además del proceso de transición y tratamientos de reasignación son algunas de las realidades a las cuales podemos acercarnos a través de la película.

Más allá de las cuestiones médicas relacionadas con la genitalidad y el intento fallido de trasplante de ovarios, nos llama la atención en la película los aspectos en la relación de pareja y del entorno social que, aunque privilegiado e idealizado, nos muestra la perplejidad y dificultad para entender y encajar a una persona, a un hombre que afirma que se siente una mujer por dentro, que "Élla siempre ha estado ahí» y, que finalmente opta por transformar su cuerpo para adaptarlo al género que siente como propio aún a costa de ser consciente de que puede costarle la vida. Es posiblemente la perplejidad a la que se enfrentan habitualmente la familia y el entorno más cercano de las personas trans y la frecuente incomprensión de una parte importante de la sociedad, lo que puede dar lugar a la discriminación e incluso a la transfobia.

\section{Otros títulos sugerentes}

El transgenerismo en el cine no siempre ha sido tratado de forma ecuánime, más bien todo lo contrario, siendo con frecuencia objeto de burla o comicidad facilona que pretende reflejar el sentir popular del machismo imperante y lo normal socialmente admitido.

Para terminar sugerimos algunos títulos que desde nuestro punto de vista pueden ser útiles para acercarnos a ésta realidad con una visión inclusiva y facilitadora de un debate respetuoso.

Mi vida en rosa / Ma vie en rose (1997) de Alain Berliner. La película muestra los problemas emocionales de una familia cuyo hijo pequeño manifiesta ser una niña, a medida que pasan por un viaje de aceptación y entendimiento.

C.R.A.Z.Y. (2005) de Jean-Mark Vallée. Centrada en las dudas de la orientación sexual en la adolescencia y juventud del personaje central y la intransigencia de un padre que repudia la homosexualidad.

Desayuno en Plutón / Breakfast on Pluto (2005) de Neil Jordan. Exquisita interpretación de Cillian Murphy, retrato desgarrador de un personaje que busca el amor y que en un entorno adverso y conservador afronta el hecho de ser diferente.

Una mujer fantástica (2017) de Sebastián Lelio. La protagonista (Daniela Vega) se enfrenta a los prejuicios de una sociedad que no entiende la cuestión del género, que la discrimina e incluso criminaliza.

Just Charlie / Just Charlie (2017) de Rebekah Fortune. Película agradable que trasmite con naturalidad todo el proceso de reasignación y de respeto hacia quien es diferente a ti. Muy apropiada para el ámbito escolar por su sencillez.

Girl / Girl (2018) de Lukas Dhont. Una historia que nos presenta el tema de la transexualidad en la adolescencia de forma natural, normalizada y con gran delicadeza.

\section{Referencias}

1. Tournier M. Celebraciones. Barcelona: El Acantilado; 2002.

2. Boletín Oficial de la Comunidad de Madrid, LEY 2/2016, de 29 de marzo, de Identidad y Expresión de Genero e Igualdad Social y no Discriminación de la Comunidad de Madrid. BOCM núm. 98 . de 26 de abril de 2016.

3. Boletín Oficial de la Comunidad de Madrid, LEY 3/2016, de 22 de julio, de Protección Integral contra la LGTBifobia y la Discriminación por Razón de Orientación e Identidad Sexual en la Comunidad de Madrid. B.O.C.M. BOCM núm. 190.

4. Becerra-Fernández A, Rodríguez-Molina JM, Asenjo-Araque N, Lucio-Pérez MJ, Cuchí-Alfaro M, García-

Rev. Med. Cine. 2020; 16(2), 111-118 Ediciones Universidad de Salamanca / @®@ J. Med. Mov., 2020; 16 (2), $111-118$ 
Camba E, et al. Prevalence, Incidence, and Sex Ratio of Transsexualism in the Autonomous Region of Madrid (Spain) According to Healthcare Demand. Arch Sex Behav. 2017;46(5):1307-12.

5. Vázquez E. La transexualidad en la obra cinematográfica de Almodóvar: «Todo sobre mi madre» Tesis doctoral. Madrid: Universidad Complutense de Madrid. Facultad de Ciencias de la Información; 2017.

6. Principios de Yogyakarta. Principios sobre la aplicación de la legislación internacional de derechos humanos en relación con la orientación sexual y la identidad de género; 2007.

7. Gómez Gil E, Esteva de Antonio I, editores. Ser transexual. Barcelona: Glosa, 2006.

8. Mas Grau J. Transexualidad y transgenerismo. Una aproximación teórica y etnográfica a dos paradigmas enfrentados. Revista de Dialectología y Tradiciones Populares 2015; LXX(2):485-501.

9. Gastó Ferrer C. Transexualidad. Aspectos Históricos y Conceptuales. C Med Psicosom. 2006;(78):13-20.

10. United Nations Development Programme (UNDP), India. Hijras / Transgender women in India: HIV, Human Rights and social exclusion. TG Issue Brief, UNDP, VC. Diciembre de 2010.

11. Guerrero I. La India despenaliza la homosexualidad. Nueva Delhi. Agencia EFE. España [Internet]. 6 de septiembre de 2018. [Citado el 20 de septiembre de 2018].
12. Newsome J. Papa Francisco pide no enseñar identidad de género en las escuelas. CNN español. [Internet]. 3 de octubre de 2016. [Citado el 22 de septiembre de 2018].

13. Zafra I. Cañizares ve una «maldad» la ley de identidad de género valenciana. El País. Comunidad Valenciana. [Internet]. 5 de enero de 2017. [Citado el 22 de septiembre de 2018].

14. Sánchez Vidal A. Esclava de nadie. Madrid: Espasa. 2010.

15. Carrillo R, Carrillo JR, Carrillo LD, Carrillo DM, CarriIlo CA. Elena de Céspedes. La azarosa vida de una cirujana del siglo XVI. Gac Med Mex. 2015;151(4):538-42.

16. Gobierno de España. Ministerio de Sanidad, Servicios Sociales e Igualdad. Clasificación Internacional de Enfermedades-10a Revisión, Modificación Clínica. $\mathrm{CIE} \cdot 10 \cdot E S$. Diagnósticos. 2a Edición. Primer tomo Madrid: Ministerio de Sanidad, Servicios Sociales e Igualdad. Secretaria General Técnica; 2018.

17. Gobierno de España. Ministerio de Sanidad, Servicios Sociales e Igualdad. Clasificación Internacional de Enfermedades-10a Revisión, Modificación Clínica. CIE·10·ES. Diagnósticos. 2a Edición. Segundo tomo Madrid: Ministerio de Sanidad, Servicios Sociales e Igualdad. Secretaria General Técnica; 2018.

18. The International Lesbian, Gay, Bisexual, Trans and Intersex Association (ILGA). [Internet]. La CIE-11 despatologiza las identidades trans y de género diverso. 24 de mayo de 2019. [Citado el 30 de mayo de 2019].

Rev. Med. Cine. 2020; 16(2), 111-118 Ediciones Universidad de Salamanca / @@ J. Med. Mov., 2020; 16 (2), 111-118 